\title{
Examining the Effect of Secondary Packaging on Microbial Penetration into Sterile Medical Device Trays
}

\author{
Ondrea Kassarjian, PhD \\ School of Packaging \\ Michigan State University, \\ East Lansing, MI, USA
}

\author{
Nora M. Bello, PhD \\ Department of Statistics, \\ Kansas State University, \\ Manhattan, KS
}

\author{
Laura Bix, PhD \\ School of Packaging \\ Michigan State University, \\ East Lansing, MI, USA
}

\author{
Gary Burgess, PhD \\ School of Packaging \\ Michigan State University, \\ East Lansing, MI, USA
}

\section{John Linz, PhD}

Food Science \& Human Nutrition

Michigan State University,

East Lansing, MI, USA

\begin{abstract}
The relative efficacy of secondary packaging on the prevention of microbial ingress into sterile medical device trays was examined.

Sterile device trays were aseptically filled with growth medium, exposed to microbial challenge, incubated, and inspected for growth. During microbial challenge, all package systems were subjected to a pressure differential that simulated those experienced during distribution.

Penetration rates were significantly decreased $(P=0.01)$ when unlidded trays were packaged in pouches (0/39), compared to those in cartons (37/39). Similarly, the number of colony forming units (CFU) present was greater for unlidded trays packaged in cartons, compared to those in pouches $(P=0.03)$. To further explore the efficacy of cartons as a barrier to microbial ingress, lidded trays with a single $100 \mu \mathrm{m}$ breach were packaged in cartons and subjected to the same methodologies; approximately 15\% exhibited growth. When compared to unlidded trays, penetration rates $(P<0.0001)$ and number of $C F U$ decreased $(P=0.0048)$.

Microbial penetration was more prevalent for sterile trays packaged within cartons than in pouches. While folding cartons are not intended to perform as sterile barriers, this suggests that microbial penetration is more likely to occur for trays packaged within cartons than those in pouches.
\end{abstract}




\section{INTRODUCTION}

The US healthcare industry continues to be one of the largest, most diverse, and fastest growing industries in the country, and healthcare spending continues to escalate. Total spending was $\$ 2.2$ trillion in 2007 , or $\$ 7,900$ per person, representing $17 \%$ of the gross domestic product (GDP). Similar rates of increase are expected over the next decade and it is estimated that by 2017 , healthcare spending will reach $\$ 4.3$ trillion, or approximately $20 \%$ of the GDP. ${ }^{1,2}$

Although many factors contribute to the rising cost of healthcare, one issue of significant concern has been the prevalence of hospital/healthcareassociated infections (HAIs) ${ }^{3,4}$ HAIs are defined as an infection that is "acquired while an individual is a patient at a hospital that was neither present nor incubating in the patient prior to receiving services in the hospital (Food and Drug Administration Amendments Act, 2007). It has been indicated that there are nearly 1.7 million HAIs annually in the US $^{3}$ which result in costs of \$5-6 billion and nearly 100,000 deaths. ${ }^{6}$ This has led to aggressive strategies to diagnose and treat infections, as well as prevent infection and transmission. ${ }^{7}$ Prevention strategies have primarily focused on the intelligent use of antimicrobials, changes in institutional policy, and improved hygiene techniques ${ }^{8,9}$

Although not frequently investigated or implicated, medical devices are also a potential source of microbes and an important risk factor for HAIs $^{10,11}$ that should be considered in prevention strategies. The use of invasive devices has been linked with the most prevalent types of HAIs. ${ }^{12}$ Hence, medical devices could serve as infection catalysts if not properly packaged to survive the rigors of distribution and handling or designed in a way that facilitates sterile technique.
Package integrity, or the ability of a package to maintain its sterile barrier throughout the distribution process, is of paramount importance in the medical device industry. ${ }^{13}$ More specifically, the question of "penetration threshold", the smallest size defect that allows microbial penetration, is of critical, practical relevance. ${ }^{14}$ An integrity test that fails packages that do not present health risks may needlessly increase costs when the package and product are destroyed unnecessarily. Conversely, integrity tests that pass packages containing breaches that allow microbial penetration constitute important health risks to patient safety. This tension of opposites is further compounded by the fact that new integrity testers are increasingly sensitive, finding smaller and smaller breaches. ${ }^{15}$ In the absence of consistent, scientifically obtained information regarding the penetration threshold, diligent companies throw out any package in which they find a breach. Recalls are made based on the potential for a breach in integrity, as opposed to a known breach of integrity. This is a conservative, but costly, approach.

Of additional interest is the fact that the primary package, the package in contact with the product (also called the sterile barrier system (SBS), is frequently packaged inside another "secondary" package (also called the protective package). The secondary packaging of medical devices is varied and commonly includes folding cartons, pouches, and wraps. In general, the purpose of secondary packaging is to provide physical protection for the SBS; however, the potential role of the secondary package as an additional barrier for microbes is unclear.

With the ultimate goal of effectively protecting patient safety, investigation of the relative efficacy of secondary packages to prevent microbial ingress 
into SBSs is warranted. Surprisingly, limited work has been done on the broad area of microbial ingress and medical device packages, ${ }^{14,}$ 16-18 and our search of the literature exposed a broad gap in knowledge regarding the effect of secondary packaging on microbial penetration.

\section{OBJECTIVE}

The objective was to conduct a comparative study examining the relative efficacy of secondary packaging (folding cartons vs. pouches) to prevent microbial ingress into sterile medical device trays.

\section{METHODS}

The study consisted of two specific questions:

Question 1: What is the relative effect of secondary package type on microbial penetration of sterile medical device trays? Tested secondary packages were:

- a solid bleached sulfate folding carton 0.015" manufactured on an Artios Kongsberg Premium Line 1930 (Figure 1a),

- and a 7.25" x 9.50" plastic pouch (100GA biaxially oriented Nylon 0.001, 0.0007 LDPE, 0.002 HDPE coex Lot \# H150978/1/A) (Mangar Industries, Inc., New Britain, PA), sealed on a SenCorp (Hyannis, MA) CeraTek Model No. 24AS/1 Serial No. 06-04236 at the following conditions: $275^{\circ} \mathrm{F}, 60 \mathrm{psi}, 1 \mathrm{~s}$ (Figure 1b).

In order to robustly test the efficacy of secondary packaging as a microbial barrier, all medical device trays used for Question 1 were left unlidded inside the secondary packages. Although unrealistic, this created an extreme condition where sterility was dependent on the secondary packaging only. Trays were randomly assigned to pouches and cartons as secondary packages (Figure 1).

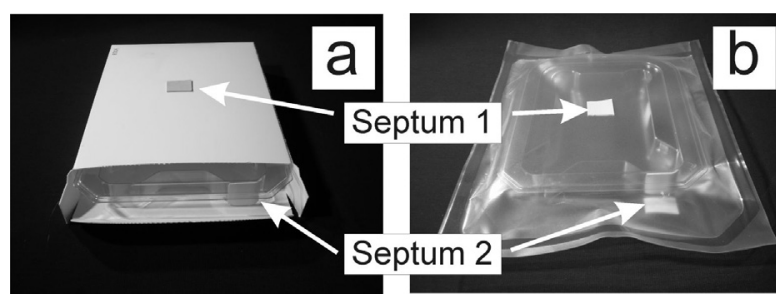

Figure 1 - Unlidded trays contained in secondary packaging, with septa: (a) carton, (b) pouch

Question 2: Given a carton as the secondary package, does the presence of a lid affect the microbial penetration of trays?

For this purpose, unlidded trays were compared with lidded trays with $100 \mu \mathrm{m}$ breaches, after both treatments had been packaged in cartons. Trays packaged individually within cartons were assigned to the absence/presence of a lid and orientation, as follows: a) unlidded trays sitting on their bottoms, and b) lidded trays sitting on their lids with bottoms facing up; tray bottoms contained a single $100 \mu \mathrm{m}$ breach. Treatment $\mathrm{b}$ was intended to represent a more realistic context for the primary packaging and, therefore, was the only treatment to contain the $100 \mu \mathrm{m}$ breach. Combinations of primary and secondary packaging (the test configurations) are henceforth referred to as "samples".

A total of 120 samples were used to investigate the specific Questions 1 and 2 of the study objective. All trays were unlidded glycol modified polyethylene terephthalate (PETG) (Perfecseal, Mankato, MN)). Forty of the samples were unlidded trays sealed in individual pouches, 40 were unlidded trays packaged individually in cartons, and the remaining 40 were lidded trays packaged individually in cartons. The latter, 
lidded trays in cartons, each contained a $100 \mu \mathrm{m}$ (+/- 10\%) thermal laser drilled pinhole (Lenox Laser, Glen Arm, MD) and were sealed with a nonporous lid. An MD2420 dual-shuttle heat sealer (Sencorp, Hyannis, MA) was used to seal the lids, LKF-002 Paper/PE/Foil/PE/HSC die cut lids (Amcor Flexibles, Madison, WI), to the trays. Batch and die positions for were recorded and sealing conditions were $300^{\circ} \mathrm{F}, 70 \mathrm{psi}, 2.5 \mathrm{~s}$. A Teflon ${ }^{\circledR}$ impregnated fiber glass cloth (Green Belting Industries, Ltd., Buffalo, NY) was used to prevent scorching and burning. All pinholes were size certified by the laser company using flow. Holes were also confirmed and characterized (post sealing) by the MSU team using a microflow detection technique manufactured by ATC, Inc. (Indianapolis, IN).

Self-sealing septa (Illinois Instruments, Johnsburg, IL) were applied to the primary packages (trays) and their secondary packages (cartons or pouches) at two locations which served as the sites for sterile growth medium injection (septum location 1) and pressure differential inducement (septum location 2) (Figure 1). For the samples with lidded trays, a septum was placed on the center of each tray lid; this eventually aligned with a septum on the carton so that, together, they served as the injection site for sterile agar (hereafter referred to as septum 1). Unlidded trays did not receive septa at this location as there was no lidstock to hold it. Additionally, a septum (hereafter referred to as septum 2) was placed in a standardized location at the end of all trays; this site served as the location for the insertion of a needle used to remove a known volume of air, inducing a pressure differential across the package (Figures 1a and 1b). After application of all septa, trays were inserted into the appropriate secondary package (pouch or carton).
Septa were also added to all secondary packages (i.e. carton or pouch) at the top center (directly above septum 1 in the case of the lidded trays), and at the end so that they aligned with septum 2 (Figures 1 and 2). Packaged trays were shipped to Smith \& Nephew, Inc. (Memphis, TN) and gamma sterilized by Sterigenics (West Memphis, AR). Packages were returned to MSU after being sterilized.

All samples were uniquely identified and aseptically injected with sterile growth medium. Just prior to filling each tray, septum 1 on the secondary package was swabbed with $70 \%$ isopropyl alcohol to prevent contamination. Trays were injected through septum 1 with molten sterile nutrient growth agar that was prepared by the Media Prep Lab at Michigan State University (East Lansing, MI). Each aliquot of agar was contained in its own stoppered bottle; this prevented the need to draw from the same growth medium multiple times, minimizing the chance for unintentional contamination. The foam bottle stoppers were swabbed with $70 \%$ isopropyl alcohol and a 60 $\mathrm{mL}$ syringe outfitted with an 18 gauge needle was pierced through each stopper to draw up 30 $\mathrm{mL}$ of agar. Then, the needle was changed to a 16 gauge vented needle, which was used to inject the agar into the packaged trays through septum 1 (Figure 1). For detailed procedures regarding microbiological techniques please reference Severin. ${ }^{14}$

Both lidded and unlidded trays were filled from the lid side; however, the orientation during filling was different. Lidded trays were oriented with their lidstock down, so that the growth medium rested on the lid and did not contact the $100 \mu \mathrm{m}$ hole that had been drilled in the bottom of the tray. Unlidded trays were filled in the opposite orientation, so that the agar rested inside the bottom of the tray. 
After the trays were filled with agar, they were loaded into an aerosolizing microbial challenge chamber built by the research team ${ }^{14}$ (Figures 2 and 3 ) in batches of four. Their orientations remained the same as when filled with agar; lidded trays sat on their lids inside the cartons so that the 100 $\mu \mathrm{m}$ holes drilled in the centers of the tray bottoms faced up and unlidded trays were positioned with the open portions of the trays facing up inside the secondary packages. Since the aerosolized spray came from the top of the chamber, these orientations were intended to induce worst-case conditions for microbial penetration in an effort to conservatively evaluate the relative performance of the treatments of interest.

Syringes used to induce pressure differential were then positioned in a racking system within the chamber. Septum 2 on the exterior of the secondary packages was swabbed with $70 \%$ isopropyl alcohol and then a syringe outfitted with an 18 gauge needles was pierced through it. This completed setup within the chamber, which was then closed.
Escherichia coli K-12 (ATCC Number 29181), a gram-negative, motile, straight-rod bacterium ranging in size from 1.1-1.5 x 2.0-6.0 $\mu \mathrm{m}$ was aerosolized using a starting concentration of $10^{\wedge} 6$ based on work conducted by Keller. ${ }^{19}$ E. coli K-12 was chosen for several reasons. It is a non-pathogenic, ubiquitous microorganism routinely used in laboratories at MSU. It is also smaller than Bacillus subtilis, the spores of which are commonly used in medical device microbial challenge studies ${ }^{15}, 20-23$ and, as a result, a more severe challenge of the sterile barrier.

For each batch of samples, the E. coli solution was aerosolized for 15 seconds and total exposure time in the chamber was 30 minutes. Pressure differential was induced simultaneously for all four trays per batch by slowly retracting the syringe racking system built into the chamber (Figures 2 and 3). The rate of air withdrawal was held constant by retracting the system over a one minute period. Pressure differential has been shown to have a significant effect on microbial penetration of these $\mathrm{e}^{14,18}$ and other similar systems..$^{24,25}$ This

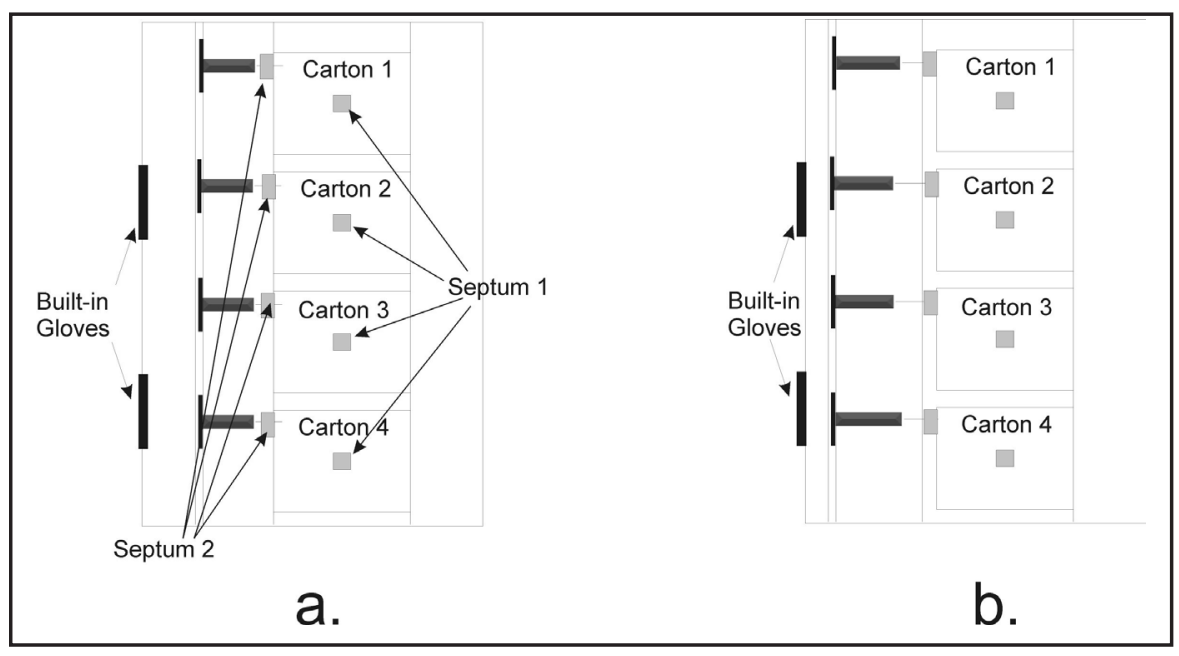

Figure 2 - Top view of aerosolization chamber when loaded with trays contained in cartons: (a) before inducement of pressure, (b) after inducement of pressure by pulling the syringe racking system utilizing built-in gloves 
is of practical relevance, as pressure differentials may be generated during the course of product distribution, such as during the descent of an aircraft or descent of a vehicle down a mountain pass. The removal of $62 \mathrm{~mL}$ of air from the package was intended to simulate a descent from an altitude of approximately $8,000 \mathrm{ft} .^{14}$
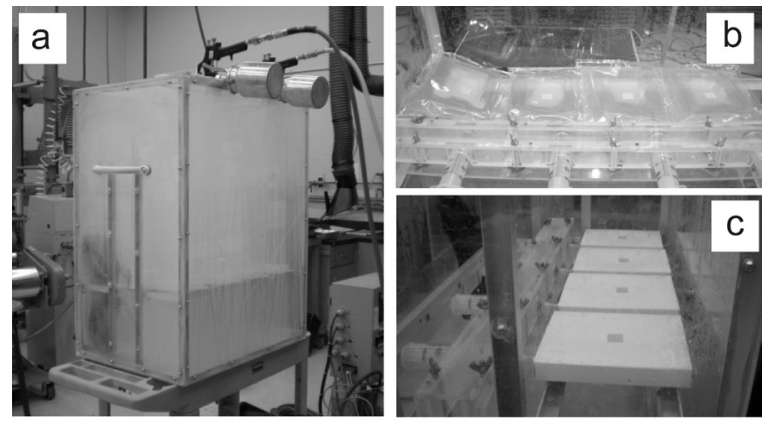

Figure 3 - Aerosolization chamber: (a) during aerosolization, (b) loaded with trays in pouches, (c) loaded with trays in cartons

After exposure in the aerosol chamber, trays were incubated at $37^{\circ} \mathrm{C}$ and $50 \% \mathrm{RH}$ for approximately 24 hours. Following the incubation period, trays were visually examined for microbial growth and colony counts were conducted (Figure 4). A single colony from each tray that exhibited growth was verified as the test organism (E. coli K-12) using an Enterotube II Identification System for Enterobacteriaceae (Becton Dickinson, San Jose, CA).

Of the 120 samples, usable data was obtained from 118. Two unlidded trays, one in a pouch and the other in a carton, were removed from the study due to technical problems associated with inadvertent puncture with the syringes.

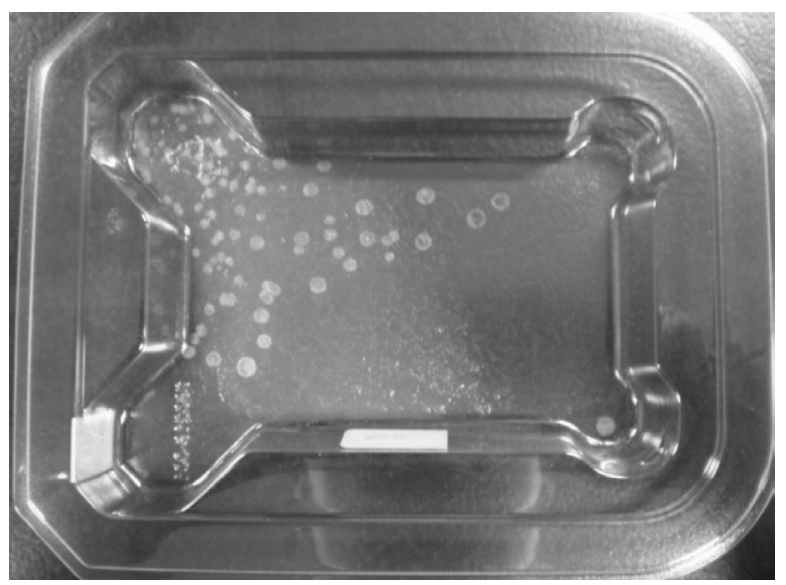

Figure 4 - Unlidded tray exhibiting growth

\section{STATISTICAL METHODS}

Microbial penetration in each tray was recorded as binary (penetration: yes/no) and count response variables (number of $\mathrm{CFU}$ ). Each response variable was modeled using a separate generalized linear mixed model assuming a binomial and an overdispersed Poisson distribution, respectively. The linear predictor in each model included the fixed effect of treatment (secondary package type or level of exposure for Questions 1 and 2, respectively), and the random effect of challenge batch nested within treatment, in order to account for technical replication present in the design. Models were fitted with the GLIMMIX procedure of SAS (SAS version 9.2, SAS Institute Inc., Cary, NC). The marginal log likelihood was approximated using an adaptive Gauss-Hermite quadrature method in order to facilitate model convergence. Due to an extreme category problem (quasi-complete separation of data points given by no penetration in any of the trays assigned to the pouch), the effect of treatment in Question 1 was modeled as a random effect in a Bayesian-type approach. Sensitivity analyses were performed using starting values for the random treatment 
variance of 1, 10, 100, 1000 and 10000. Variance estimates and treatment differences were not affected by the starting values considered, thus the proposed Bayesian analysis was considered robust and used for inference. Contrasts were used to compare treatments. Estimated least square means and standard errors of the probability of penetration, as well as CFU, were reported.

\section{RESULTS}

Question 1: What is the relative effect of secondary package type on microbial penetration of sterile medical device trays?

None of the unlidded trays in pouches exhibited microbial growth. In contrast, most of the unlidded trays in cartons did $(n=39)$.

Secondary packaging was identified to have a significant effect on the probability of microbial penetration when the contained medical device trays were unlidded ( $\mathrm{P}=0.01)$; microbial penetration was more likely for trays packaged within cartons than for those within pouches (Figure 5a). The number of CFU per unlidded tray was also significantly greater when packed within cartons than within pouches $(\mathrm{P}=0.03)$ (Figure
$5 b)$.

Question 2: Given a carton as the secondary package, does the presence of a lid affect the microbial penetration of trays?

Level of exposure (lidded or unlidded) affected the probability of microbial penetration into the trays packaged within cartons $(\mathrm{P}<0.0001)$. Microbial penetration of medical device trays inside cartons was more likely to occur with unlidded trays compared to lidded (Figure 6a). In addition, the presence or absence of a lid had a significant effect on the number of $\mathrm{CFU}$ $(\mathrm{P}=0.0048)$, such that the number of $\mathrm{CFU}$ that entered trays within cartons was greater when they were unlidded compared to lidded (Figure 6b).

\section{DISCUSSION}

This study suggests differences in both the rates of contamination and number of $\mathrm{CFU}$ that penetrated trays when pouches were compared with cartons as secondary packages. The pouches outperformed the cartons as effective secondary packaging barriers to microbial ingress, as shown with the rates of penetration and number of CFU.

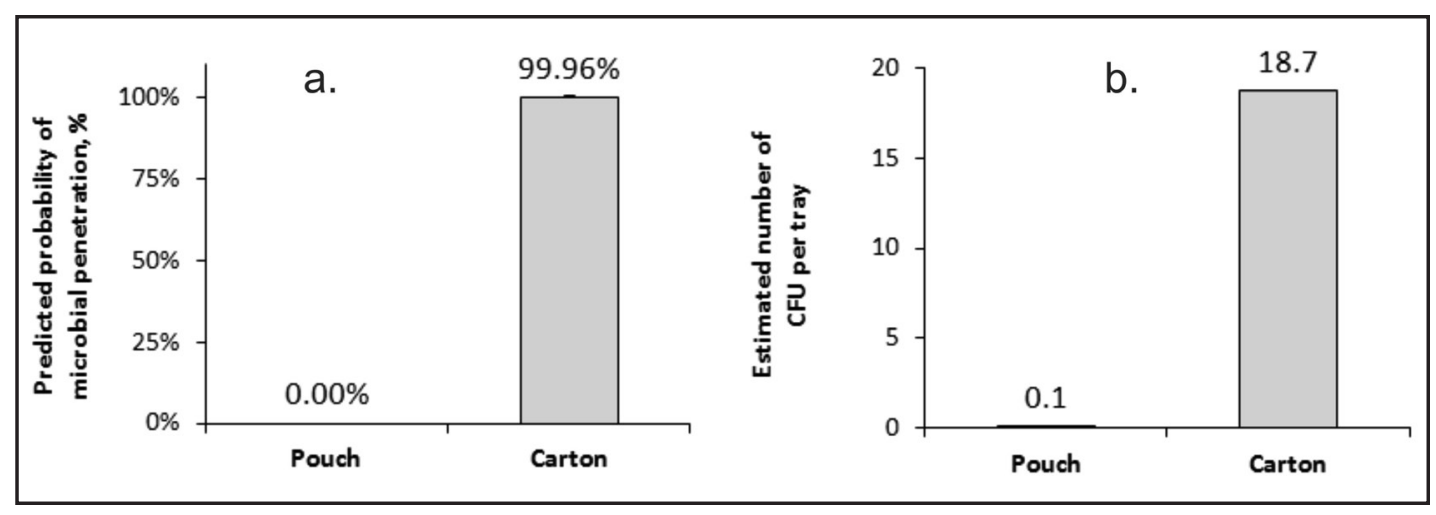

Figure 5 - Estimated least square means and standard errors for: (a) probability of microbial penetration, and (b) number of CFU for unlidded trays in pouches and cartons 


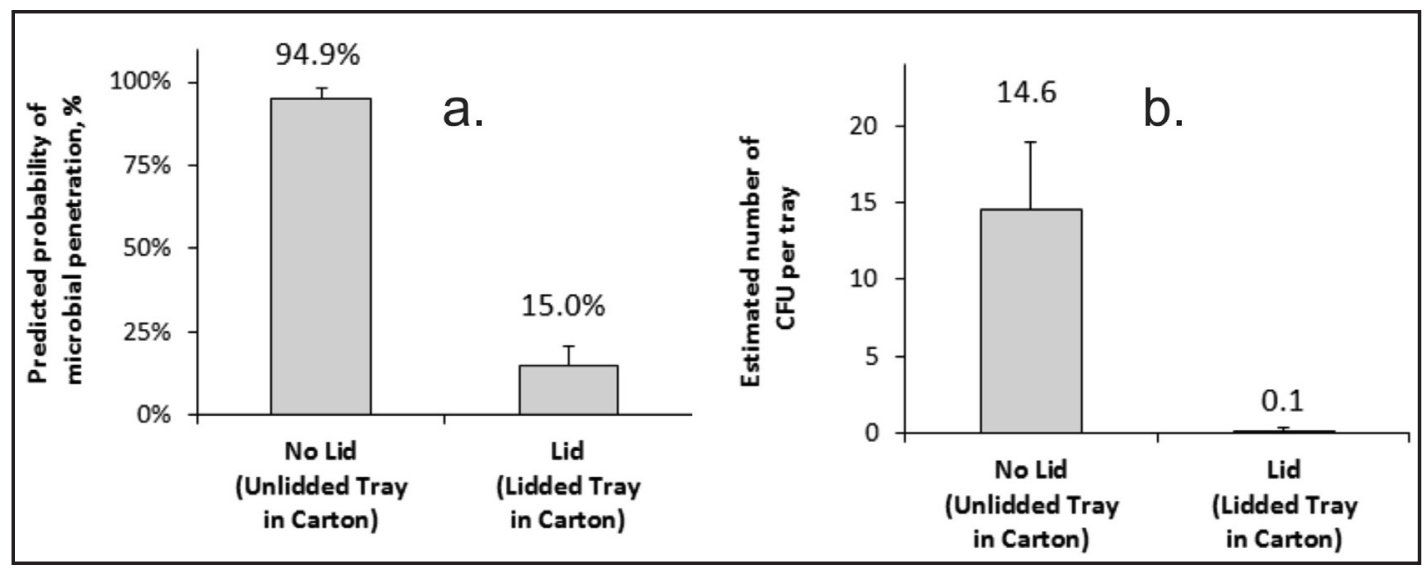

Figure 6 - Estimated least square means and standard errors of (a) probability of microbial penetration, and (b) number of CFU for unlidded and lidded trays in cartons

These results are not surprising given the sealed structure of the pouch, which contrasts with the presence of unsealed crevices of the carton. It is also notable that the microbial challenge conditions used were intended to be substantially more extreme than normal expected conditions of shipment.

We acknowledge that unlidded trays are not realistic in a medical setting; however, they were used in this study as a control to assess microbial ingress through secondary packages into device trays presented under commercial conditions, as represented by the lidded treatment for Question 2. The lidded trays with pinhole defects experienced microbial penetration in $15 \%(6 / 40)$ of the tested trays despite the presence of the carton.

Packaged medical devices have to survive sterilization, shipping, and handling with their sterile barrier systems (SBSs) intact. Packaging manufacturers and the device companies that employ them do not consider secondary packaging as part of the SBS or purport that they provide microbial barrier. ${ }^{13}$ Other benefits and determents of secondary packaging systems also come into consideration when making a packaging decision, such as stacking capabilities, billboard, barrier, and cost, among others.

Results presented in this study have direct implications for patient safety. Due to high rates of nosocomial infections, $5,9,26$ package integrity (the ability of a package to maintain its sterile barrier) is imperative. At the same time, a reduction in the cost of healthcare is viewed as a national, if not international, need. ${ }^{1,2}$ Understanding the precise conditions in which microbial penetration occurs and the level of benefit (protection, identification, convenience, etc.) packaging systems afford is of paramount importance. The rates and treatment challenges of healthcare-associated infections, and the potential for devices to serve as reservoirs for microorganisms, make further study of this issue imperative.

\section{LIMITATIONS}

The self-built tailored-approach used to address the research questions had logistical limitations 
that caused concerns over the experimental design. Specifically, packaged trays grouped in batches of four were tested in a sequential manner: first unlidded trays within pouches, then unlidded trays within cartons, and finally, lidded trays within cartons. This sequential order of treatment evaluation was due to a logistical limitation of physical adjustments that had to be made to the racking system of the aerosolizing chamber at each treatment switch. We acknowledge the potential for an ordering effect to be partially confounded with the reported treatment effects.

\section{ACKNOWLEDGEMENTS}

\section{Financial support.}

The authors wish to acknowledge: Abbott Vascular; ATC, Inc.; Cardinal Health Medical Products and Services; DePuy Orthopaedics, Inc; DDL; Ethicon and Ethicon Endosurgery; Flexible Packaging Association; Medtronic; Smiths Medical, Critical Care; and Smith \& Nephew. Additional insights, influence, and assistance provided by Dennis Young, Ashley Rudy, Ken Gruhl, Hal Miller, John Spitzley and Curt Larsen are greatly appreciated. The authors wish to acknowledge ATC, Inc., Lansmont, Mangar, and Smith \& Nephew for in-kind donations.

\section{Potential conflicts of interest.}

Research was funded and supported from a variety of industry sources disclosed in the acknowledgements; these include Mangar, the company that provided the pouches.

\section{REFERENCES}

[1] Keehan S, Sisko A, Truffer C, Smith S, Cowan C, Poisal J, et al. Health Spending Projections Through 2017: The Baby-Boom Generation is Coming to Medicare. Health
Aff 2008;27:145-55. Link

[2] National Coalition on Health Care. Facts on Health Care Costs. 2009.

[3] Stone PW, Larson E, Kawar LN. A systematic audit of economic evidence linking nosocomial infections and infection control interventions: 1990-2000. Am J Infect Control 2002;20:145-52. Link

[4] Stone PW, Braccia D, Larson E. Systematic review of economic analysis of health careassociated infections. Am J Infect Control 2005;33:501-9. Link

[5] Klevens RM, Edwards JR, Richards CL, Horan TC, Gaynes RP, Pollock DA, et al. Estimating Health Care-Associated Infections and Deaths in U.S. Hospitals, 2002. Public Health Rep 2007;122:160-6. Link

[6] Murphy D, Whiting J, Hollenbeak CS. Dispelling the Myths: The True Cost of Healthcare-Associated Infections. Association for Professionals in Infection Control and Epidemiology. 2007;1-15. Link

[7] Notice to Readers: CDC's Campaign to Prevent Antimicrobial Resistance in Health-Care Settings. Centers for Disease Control and Prevention website. http:// www.cdc.gov/mmwr/preview $/ \mathrm{mmwrhtml} /$ mm5115a5.htm. Published 2002. Accessed April 7, 2011.

[8] Harbath S, Sax H, Gastmeier P. The preventable proportion of nosocomial infections: an overview of published reports. J Hosp Infect 2003;54:258-66. Link

[9] Malone N, Larson E. Factors associated with a significant reduction in hospitalwide infection rates. Am J Infect Control 1996;24:180-5. Link

[10] Schierholz JM, Beuth J. Implant infections: a haven for opportunistic bacteria. J Hosp Infect 2001;49:87-93. Link

[11] Jarvis WR. Selected Aspects of the 
Socioeconomic Impact of Nosocomial Infections: Morbidity, Mortality, Cost, and Prevention. Infect Control Hosp Epidemiol 1996;17:552-7. Link

[12] Richards MJ, Edwards JR, Culver DH, Gaynes RP. Nosocomial Infections in Combined Medical-Surgical Intensive Care Units in the United States. Infect Control Hosp Epidemiol 2000;21:510-5. Link

[13] Association for the Advancement of Medical Instrumentation. ANSI/AAMI/ISO 116071:2006 Packaging for terminally sterilized medical devices - Part 1: Requirements for materials, sterile barrier systems, and packaging systems. Association for the Advancement of Medical Instrumentation, 1110 N. Glebe Road, Suite 220, Arlington, VA 22201-4795 United States; 2006.

[14] Severin J. The Effect of Pressure Differential on Microbial Penetration of a Sterile Medical Device Tray. East Lansing: School of Packaging, Michigan State University; 2006.

[15] Jones L, Hansen J, Anderson H, Larsen C, Miller H, Scholla M, et al. In Quest of Sterile Packaging: Part 2 Physical Package Integrity Test Methods. Medical Device \& Diagnostic Industry 1995;17:81-5.

[16] Dunkelberg H, Wedekind S. Preliminary Results for a New Final Package Test to Assess the Quality of Sterile Package Systems. Infect Control Hosp Epidemiol 2004;25:26-9. Link

[17] Dunkelberg H, Schmelz U. Determination of the Efficacy of Sterile Barrier Systems Against Microbial Challenges During Transport and Storage. Infect Control Hosp Epidemiol 2009;30:179-83. Link

[18] Severin J, Bix L, Gilliland D, Lockhart H. A New Methodology for Whole-Package Microbial Challenge Testing for Medical
Device Trays. Journal of Testing and Evaluation 2007;35:373-80. Link

[19] Keller SW, Marcy JE, Blakistone BA, Lacy GH, Hackney CR, Carter WH. Bioaerosol Exposure Method for Package Integrity Testing. Journal of Food Protection 1996;59:768-71. Link

[20] Reich R. A Method for Evaluating the Microbial Barrier Properties of Intact Packages. Medical Device \& Diagnostic Industry 1985;7:80-88.

[21] Hansen J, Jones L, Anderson H, Larsen C, Miller H, Scholla M, et al. In Quest of Sterile Packaging: Part 1 Approaches to Package Testing. Medical Device \& Diagnostic Industry 1995; 17:56-61.

[22] ASTM. F1608-00 Standard Test Method for Microbial Ranking of Porous Packaging Materials (Exposure Chamber Method). ASTM International, 100 Barr Harbor Drive, PO Box C700, West Conshohocken, PA 19428-2959 United States; 2009.

[23] Placencia AM, Oxborrow GS, Peeler JT. Package Integrity Methodology for Testing Biobarrier Properties of Porous Packaging, Part II: FDA Exposure Chamber Method. Medical Device \& Diagnostic Industry 1986;8:46-53.

[24] Dunkelberg H, Fleitmann-Glende F. Measurement of the microbial barrier effectiveness of sterilization containers in terms of the $\log$ reduction value for prevention of nosocomial infections. Am J Infect Control 2006;34:285-9. Link

[25] Dunkelberg H, Rohmann S. Test to Determine Sterile Integrity of Wrapped Medical Products at a Probability of Recontamination of $1: 1,000,000$. Infect Control Hosp Epidemiol 2006;27:367-71.

[26] CDC NNIS System. National Nosocomial Infections Surveillance (NNIS) System 
Report, data summary from January 1992

through June 2004, issued October 2004.

Am J Infect Control 2004;32: 470-85. 